Check for updates

Cite this: RSC Adv., 2021, 11, 17283

Received 6th December 2020

Accepted 4th May 2021

DOI: $10.1039 /$ dOra10281d

rsc.li/rsc-advances

\section{Dual-emission ratio fluorescence for selective and sensitive detection of ferric ions and ascorbic acid based on one-pot synthesis of glutathione protected gold nanoclusters $\uparrow$}

\author{
Shuai Zhang, ${ }^{a}$ Cong Zhang, ${ }^{a}$ Xiaodong Shao, ${ }^{b}$ Rentian Guan, ${ }^{a}$ Yingying Hu, ${ }^{a}$ \\ Keying Zhang, ${ }^{a}$ Wenjing Liu, ${ }^{a}$ Min Hong (D) a and Qiaoli Yue (iD *a
}

\begin{abstract}
A fluorometric method was proposed for the determination of $\mathrm{Fe}^{3+}$ and ascorbic acid (AA) based on blue and red dual fluorescence emissions of glutathione (GSH) stabilized-gold nanoclusters (AuNCs). AuNCs were synthesized from GSH and tetrachloroauric acid. The fluorescence peaks of AuNCs were at $425 \mathrm{~nm}$ and $585 \mathrm{~nm}$, respectively. In the presence of $\mathrm{Fe}^{3+}$, the fluorescence peak at $425 \mathrm{~nm}$ can be enhanced and that at $585 \mathrm{~nm}$ can be quenched. There is a good linear relationship between the fluorescence intensity ratio for the 425 and $585 \mathrm{~nm}$ peaks $\left(F_{425} / F_{585}\right)$ and the concentration of $\mathrm{Fe}^{3+}$ in the range of 0.75-125 $\mu \mathrm{M}$. However, when AA was added to the AuNCs-Fe ${ }^{3+}$ system, the value of $F_{425} / F_{585}$ decreased consistently with the concentration of $A A$ in the range of $0.25-35 \mu \mathrm{M}$. The limit of detection for $\mathrm{Fe}^{3+}$ and $\mathrm{AA}$ was 227 and $75.8 \mathrm{nM}$, respectively. The interaction between AuNCs and $\mathrm{Fe}^{3+}$ can induce the ligand-metal charge transfer (LMCT) effect leading to the fluorescence increment at $425 \mathrm{~nm}$, while $\mathrm{AA}$ can reduce $\mathrm{Fe}^{3+}$ to $\mathrm{Fe}^{2+}$. The production of $\mathrm{Fe}^{2+}$ can not enhance or quench the fluorescence of AuNCs. By comparison with previous literature, the AuNCs prepared here show two fluorescence peaks without additional fluorescence labels. Furthermore, the method was successfully applied in the determination of $\mathrm{Fe}^{3+}$ and $\mathrm{AA}$ in some real samples, such as water, human serum and tablets.
\end{abstract}

\section{Introduction}

As important metal ions in the biological system, iron ions $\left(\mathrm{Fe}^{3+}\right)$ play an important role in physiological and pathological processes such as cell metabolism, oxygen transport, enzyme catalysis, electron transfer and RNA synthesis. ${ }^{\mathbf{1 , 2}}$ In general, iron deficiency leads to anemia, hypotension, and weakened immunity. ${ }^{3}$ In contrast, excessive intake of $\mathrm{Fe}^{3+}$ will lead to the production of highly reactive oxygen species, thus damaging nucleic acids, lipids and proteins in humans and animals. ${ }^{4}$ In addition, the cytotoxicity of $\mathrm{Fe}^{3+}$ may cause some diseases, such as Parkinson's disease and Alzheimer's disease. ${ }^{5}$ Therefore, the accurate determination of $\mathrm{Fe}^{3+}$ is of great significance in health and environmental monitoring.

Ascorbic acid (vitamin C, AA) is an important micronutrient, antioxidant, and neurochemical. It plays a key role in various

${ }^{a}$ Shandong Provincial Key Laboratory of Chrmical Energy Storage and Novel Cell Technology, School of Chemistry and Chemical Engineering, Liaocheng University, China.E-mail: yueqiaoli@yahoo.com

${ }^{b}$ Nanobioengineering/Bioelectronics Laboratory, Department of Biomedical Engineering, Florida International University, Miami, FL 33174, USA. E-mail: licz@ fiu.edu

$\dagger$ Electronic supplementary information (ESI) available. See DOI: 10.1039/d0ra10281d oxidative stress-related diseases such as Parkinson's disease, Alzheimer's disease, cardiovascular disease and some types of cancer. $^{6-10}$ Studies have shown that AA can reduce oxidative stress generated by metal ions in neurons, thereby inhibiting neurodegeneration induced by mitochondrial autophagy. ${ }^{\mathbf{1 1}} \mathrm{AA}$ provides neuroprotection in brain injury/dysfunction, regulates nerve function, removes free radicals from cells, and ACTS as an anti-scurvy agent. ${ }^{6,12,13}$ Therefore, a rapid, sensitive and selective detection of AA in biological fluids is still an active topic in biosensor research and in various fields such as cell biology, drug screening and medical diagnosis. ${ }^{9}$

The traditional metal ion detection methods include atomic absorption spectrometry, inductively coupled plasma atomic emission spectrometry, voltammetry, extraction spectrophotometry, resonance light scattering spectrometry, etc. ${ }^{\mathbf{1 4 - 1 6}}$ The detection methods for AA include chromatography, capillary electrophoresis, colorimetry, electrochemical methods, etc. $^{{ }^{6-9}}$ Due to its high sensitivity and easy operation, fluorescence spectrometry is becoming increasingly important in quantitative analysis. Some nanomaterials and organic molecules can be used as fluorescent probes to detect $\mathrm{Fe}^{3+}$ and AA. ${ }^{17-22}$ However, these probes are usually difficult to prepare.

As a new type of luminescent nanomaterials, metal nanoclusters have attracted much attention in recent years in the 
fields of chemistry, biology, materials science and so on. Fluorescent AuNCs are widely used in sensing, biomarkers and bioimaging due to their small size, ease of preparation, good biocompatibility and high fluorescence. In particular, AuNCs with GSH as a template have the advantages of high fluorescence intensity and obvious emission peak, and the emission peak is basically after $550 \mathrm{~nm}$. In this case, the corresponding fluorescent substance can be added to AuNCs system as a marker to form a dual-emission system. ${ }^{23-26}$ These fluorescent materials usually include metal organic framework compounds (MOFs), carbon dots (CDs) and some fluorescent organic compounds, which usually show blue emission. The composite materials form a "blue-red" double emissions system, and the ratio-type AuNCs probes can be developed.

In this work, we synthesized a new type of red fluorescence AuNCs with GSH as template and stabilizer. AuNCs showed double fluorescence peaks without the addition of fluorescence markers, which is different from the above methods. Thus the influence of some species can be effectively eliminated, for example, the common $\mathrm{Hg}^{2+}$ quenching effect on the fluorescence of AuNCs. Furthermore, the AuNCs were used for the detection of $\mathrm{Fe}^{3+}$ and $\mathrm{AA}$ with high sensitivity and selectivity.

\section{Experimental section}

\section{Materials and reagents}

GSH, AA and $\mathrm{HAuCl}_{4} \cdot 3 \mathrm{H}_{2} \mathrm{O}$ were ordered from Shanghai Perfemiker Material Tech Co., Ltd (Shanghai, China, http:// www.canspecsci.com). Amino acids and other organic species were obtained from Beijing Solarbio Science \& Technology Co., Ltd (Beijing, China, http://www.solarbio.com). There were Lcysteine (Cys), glutamic acid (Glu), proline (Pro), methionine (Met), L-aspartic acid (Asp), glycine (Gly) and melamine (Mel), and $o$-phenanthroline, $o$-phenylenediamine, urea, and uric acid (UA). Acid, base and some common salts were purchased from Aladdin Reagent Co., Ltd (Shanghai, China). They included $\mathrm{HCl}$, $\mathrm{HAc}, \mathrm{NaOH}, \mathrm{NaCl}$, quinine sulphate, $\mathrm{NaH}_{2} \mathrm{PO}_{4}, \mathrm{Na}_{2} \mathrm{HPO}_{4}, \mathrm{NaAc}$, and $\mathrm{FeNO}_{3}, \mathrm{FeSO}_{4}, \mathrm{CuCl}_{2}, \mathrm{HgCl}_{2}, \mathrm{Zn}\left(\mathrm{CH}_{3} \mathrm{COO}\right)_{2}, \mathrm{Mn}\left(\mathrm{NO}_{3}\right)_{2}$, $\mathrm{Pb}\left(\mathrm{NO}_{3}\right)_{2}, \mathrm{MgCl}_{2}, \mathrm{CoCl}_{2}, \mathrm{BaCl}_{2}, \mathrm{CrCl}_{3}, \mathrm{NaCl}, \mathrm{NiSO}_{4} \cdot 6 \mathrm{H}_{2} \mathrm{O}$, $\mathrm{CdCl}_{2}$, and $\mathrm{CaCl}_{2}$. All chemicals from commercial sources were of analytical grade and used directly without further purification if no special instructions. All solutions were prepared and diluted by ultrapure water from Milli-Q water purified system.

\section{Instruments}

Fluorescence spectra were obtained on an F-7000 spectrophotometer (Hitachi, Japan). Absorption spectral measurements were carried out on a UV-2550 ultraviolet spectrophotometer (Shimadzu, Japan). Transmission electron microscope (TEM) data were performed on a JEM 2100 electron microscope (JEOL Ltd, Japan). TEM worked with an acceleration voltage at $200 \mathrm{kV}$, and a carbon-coated copper grid was used for sample suspension. Fourier Transform infrared (FT-IR) spectra were carried out on a Nicolet 6700 FT-IR spectrophotometer (Thermo Scientific Ltd, USA). X-ray photoelectron spectroscopy (XPS) measurements were undertaken with a K-Alpha spectrometer
(Thermo Scientific Ltd, USA). Digital photos of AuNCs solution under UV lamp illumination were captured by a Coolpix 4500 digital camera.

\section{Preparation of AuNCs}

AuNCs were prepared using a hydrothermal method by referencing to previous method with some changes. ${ }^{27}$ GSH was employed for the reduction and stabilization of AuNCs. Before use, all glasswares were washed carefully with Aqua Regia. $10 \mathrm{~mL} \mathrm{HAuCl}_{4}(20 \mathrm{mM})$ was diluted with $87 \mathrm{~mL}$ deionized water, at room temperature. $3 \mathrm{~mL} \mathrm{GSH}$ solution $(100 \mathrm{mM})$ was added to the $\mathrm{HAuCl}_{4}$ solution under moderate magnetic stirring. The mixture of $\mathrm{GSH}$ and $\mathrm{HAuCl}_{4}$ was heated gradually to $80^{\circ} \mathrm{C}$ with continuous stirring for 15 hours. It indicated the formation of AuNCs with red fluorescence when the transparent solution gradually changed to golden yellow solution. After cooling to room temperature, the AuNCs products were obtained and stored at $4{ }^{\circ} \mathrm{C}$ before use.

\section{Determination of $\mathrm{Fe}^{3+}$ and $\mathrm{AA}$}

Determination of $\mathrm{Fe}^{3+}$ or AA was performed in aqueous solution at room temperature. Firstly, standard stock solutions with different concentrations were prepared by dissolving $\mathrm{Fe}^{3+}$ or AA in deionized water. Then $0.5 \mathrm{~mL}$ AuNCs was added to $1.5 \mathrm{~mL}$ microcentrifuge tube and diluted to $1 \mathrm{~mL}$. Then $\mathrm{Fe}^{3+}$ solutions with different concentrations were added and mixed in a vortex mixer for $10 \mathrm{~min}$. The fluorescence spectra of AuNCs for the obtained solutions (Ex $390 \mathrm{~nm}$ ) were recorded in the presence and absence of $\mathrm{Fe}^{3+}$, respectively. Subsequently, AA was detected in aqueous solution at room temperature. A series of AA solutions were added and mixed with AuNCs- $\mathrm{Fe}^{3+}$ solution in a vortex mixer for $10 \mathrm{~min}$. The fluorescence spectra of AuNCs$\mathrm{Fe}^{3+}$ (Ex $\left.390 \mathrm{~nm}\right)$ were recorded with and without AA, respectively.

\section{Pretreatment of real samples}

Tap water samples were collected from the laboratory of Department of Chemistry at Liaocheng University. Lake water samples were obtained from the artificial lake inside the campus of Liaocheng University. After all water samples were settled overnight, the supernatant was diluted with ultra-pure water. The fetal bovine serum samples were diluted directly with ultra-pure water, and the standard solutions of $\mathrm{Fe}^{3+}$ with different concentrations were spiked. VC tablets were grounded into fine powder, and dissolved in ultra-pure water. The supernatant was diluted with ultra-pure water. In order to verify the accuracy of the method, $\mathrm{Fe}^{3+}$ and $\mathrm{AA}$ in the same samples were simultaneously determined by the national standard methods. ${ }^{28}$

\section{Results and discussion}

\section{Design strategy}

The assay strategy for the determination of $\mathrm{Fe}^{3+}$ and AA was shown in Scheme 1. Firstly, AuNCs with blue and red fluorescence emissions were synthesized with GSH, which was for the 


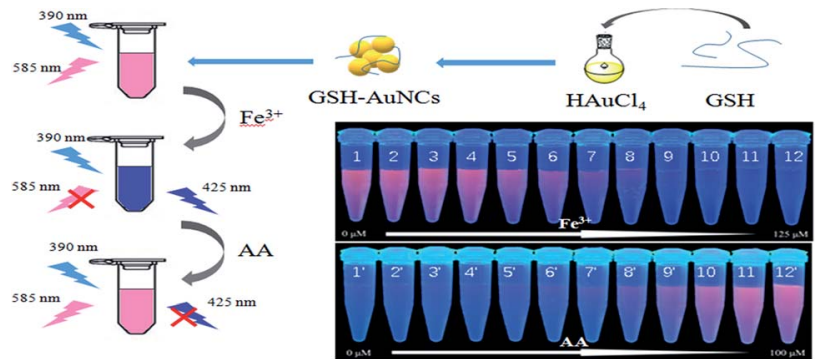

Scheme 1 Schematic illustration for synthesis of AuNCs and their application in the determination of $\mathrm{Fe}^{3+}$ and $\mathrm{AA}$.

reduction and stabilization of AuNCs. Under excitation of $390 \mathrm{~nm}$, AuNCs showed two fluorescence peaks at 425 and $585 \mathrm{~nm}$, respectively. The fluorescence intensity at $585 \mathrm{~nm}$ can be quenched by $\mathrm{Fe}^{3+}$, while that of $425 \mathrm{~nm}$ can be enhanced simultaneously. Furthermore, the fluorescence ratio at 425 and $585 \mathrm{~nm}\left(F_{425} / F_{585}\right)$ increased gradually with the concentration of $\mathrm{Fe}^{3+}$ increase. Thus, the fluorescence of AuNCs can be used to determine $\mathrm{Fe}^{3+}$. When AA was added to the AuNCs- $\mathrm{Fe}^{3+}$ system, however, the fluorescence of AuNCs was recovered gradually. And then the AuNCs- $\mathrm{Fe}^{3+}$ system can be employed for the determination of AA.

\section{Characterization of GSH-AuNCs with and without analytes}

Optical properties. Template synthesis of AuNCs is shown in Scheme 1 . As previously reported, ${ }^{27}$ AuNCs were synthesized by a simple and environmentally friendly method. Firstly, the optical properties of AuNCs were studied using the UV-vis absorption and fluorescence spectrophotometry. As shown in Fig. 1a, there is an obvious absorption peak at $390 \mathrm{~nm}$. AuNCs show bright red fluorescence under $365 \mathrm{~nm}$ UV light (Fig. 1a inset). It can be further confirmed by fluorescence spectra that the excitation and emission peaks are at $390 \mathrm{~nm}$ and 425, $585 \mathrm{~nm}$, respectively (Fig. 1a). In addition, to obtain the optimum excitation wavelength, the effect of excitation wavelength on the emission intensity was investigated. It can be observed from Fig. $1 \mathrm{~b}$ that there are two emission peaks under all excitation lights. The emission peak intensity increases with excitation wavelength from $360 \mathrm{~nm}$ to $390 \mathrm{~nm}$, and decreases over $390 \mathrm{~nm}$. Thus, the maximum excitation wavelength is selected at $390 \mathrm{~nm}$.

It can be clearly observed that there are double emission peaks at $425 \mathrm{~nm}$ and $585 \mathrm{~nm}$ in the fluorescence spectra of AuNCs, respectively (Fig. S1, in ESI $\dagger$ ). In the presence of $\mathrm{Fe}^{3+}$, the fluorescence intensity at $585 \mathrm{~nm}$ is quenched, and that of $425 \mathrm{~nm}$ is enhanced. When AA was added to the system of AuNCs- $\mathrm{Fe}^{3+}$, the fluorescence for AuNCs was recovered at 585 and $425 \mathrm{~nm}$. Moreover, the fluorescence change of AuNCs induced by AA can be ignored completely. It can be deduced that the fluorescence recovery for AuNCs induced by AA can be ascribed to the interaction between $\mathrm{Fe}^{3+}$ and AA.

TEM images. The morphology of AuNCs was investigated with and without $\mathrm{Fe}^{3+}$ or AA using TEM (Fig. 2). AuNCs show sphere and monodisperse without obvious aggregation (Fig. 2a) with an average size at $1.4 \pm 0.6 \mathrm{~nm}$ (Fig. 2b). The size
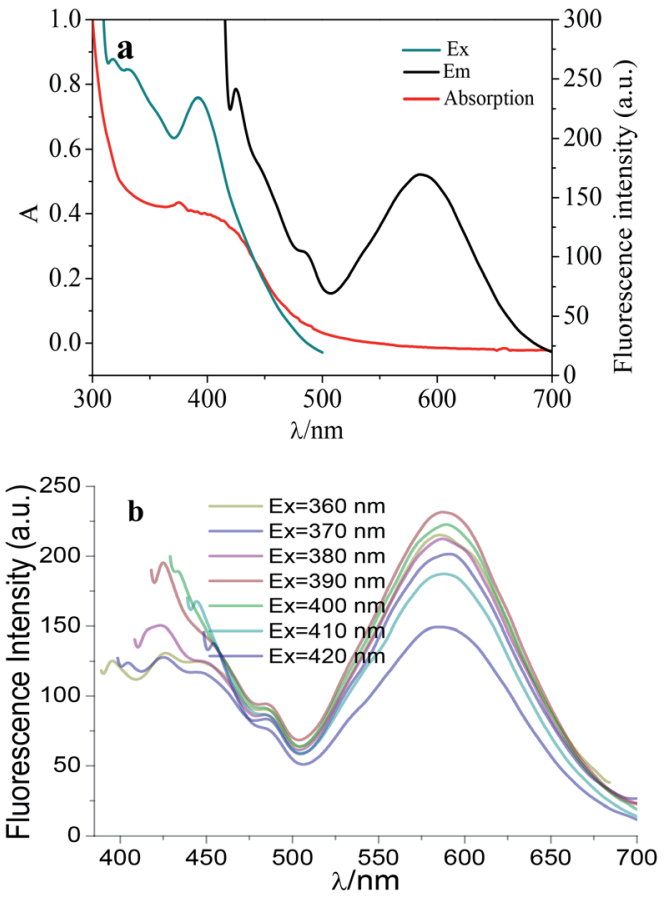

Fig. 1 The absorption and fluorescence spectra of AuNCs (a) and the effect of excitation wavelength on the emission spectrum of AuNCs (b). The emission and excitation wavelength were fixed at $585 \mathrm{~nm}$ (a) and $390 \mathrm{~nm}$ (b), respectively.

distribution of $51 \%$ AuNCs are observed at the range of $2.0-$ $2.2 \mathrm{~nm}$. In the presence of $\mathrm{Fe}^{3+}$, AuNCs display obvious aggregation (Fig. 2c), with an average size of $5.7 \pm 1.8 \mathrm{~nm}$ (Fig. 2d). While in the presence of both $\mathrm{Fe}^{3+}$ and AA, AuNCs exhibit almost monodisperse again (Fig. 2e). The size distribution ranges from $1.5 \mathrm{~nm}$ to $2.6 \mathrm{~nm}$ and $67 \%$ of AuNCs are in the range of 2.0-2.2 nm (Fig. 2f). It suggests that the assembled AuNCs induced by $\mathrm{Fe}^{3+}$ are redispersed with the addition of AA.

XPS. In order to study the composition of AuNCs, the valence state of $\mathrm{Au}$ in AuNCs structure was determined by HR-XPS. There are two peaks in the high resolution XPS of AuNCs at $84.1 \mathrm{eV}$ and $87.7 \mathrm{eV}$, respectively (Fig. 3a). They correspond to the binding energies of $4 f_{7 / 2}$ and $4 f_{5 / 2}$ of $A u$, respectively. It is proved that there are two valence states of $\mathrm{Au}(0)$ and $\mathrm{Au}(\mathrm{I})$ in AuNCs. Furthermore, the peak of $\mathrm{Au} 4 \mathrm{f}_{7 / 2}$ can be further deconvoluted into two distinct peaks with binding energies of 83.68 and $84.20 \mathrm{eV}$, while the peak of $A u 4 f_{5 / 2}$ can be divided by deconvolution into two different peaks of 87.39 and $87.88 \mathrm{eV}$, respectively. The results are consistent with the previous work. ${ }^{29}$ For the co-existence of $\mathrm{Au}(0)$ and $\mathrm{Au}(\mathrm{I})$, it indicates that $\mathrm{Au}(0)$ exists in the core of AuNCs, and $\mathrm{Au}(\mathrm{I})$ is in the complex of GSH$\mathrm{Au}$ on the surface of AuNCs, which leads to a high fluorescence property of AuNCs. ${ }^{30}$ When $\mathrm{Fe}^{3+}$ was added to the solution of AuNCs, the two binding energies for XPS peaks of $4 \mathrm{f}_{7 / 2}$ and $4 \mathrm{f}_{5 / 2}$ for Au were as similar as those for AuNCs (Fig. 3b). The peaks of both $4 \mathrm{f}_{7 / 2}$ and $4 \mathrm{f}_{5 / 2}$ for AuNCs- $\mathrm{Fe}^{3+}$ divided by deconvolution are different from those for AuNCs. By comparison, the peaks at 83.36 and $87.39 \mathrm{eV}$ in AuNCs-Fe ${ }^{3+}$ system are higher than those of AuNCs. It can be suggested that $\mathrm{Fe}^{3+}$ can induce the change of 

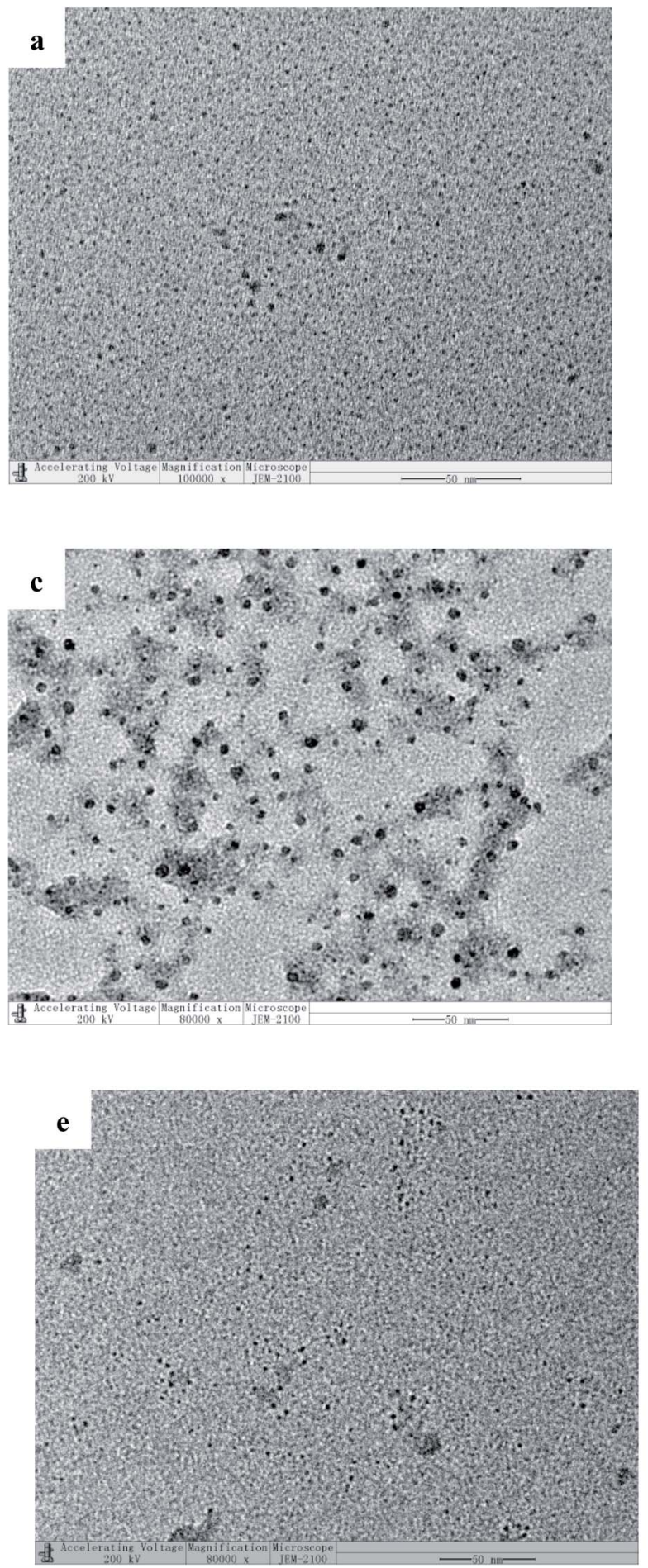
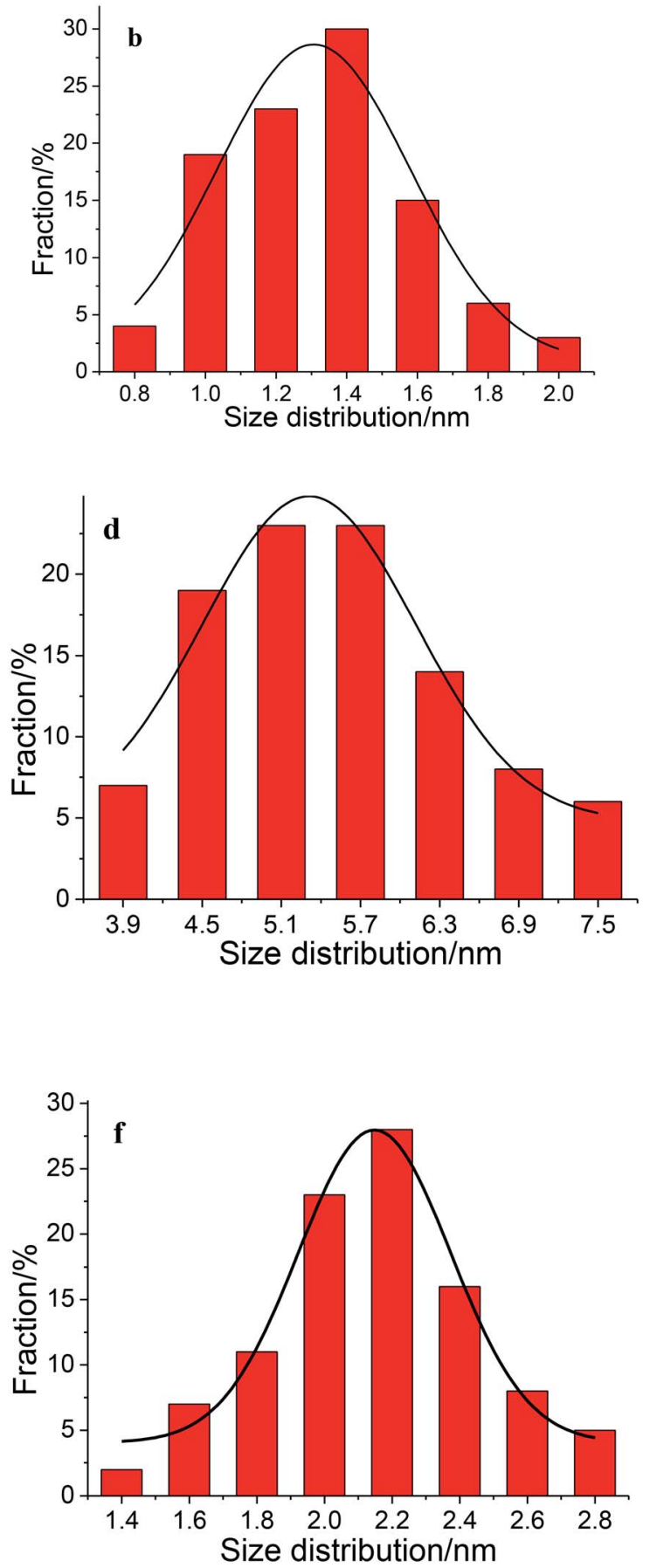

Fig. 2 TEM images of AuNCs (a), AuNCs $+\mathrm{Fe}^{3+}$ (c), and AuNCs $+\mathrm{Fe}^{3+}+\mathrm{AA}(\mathrm{e})$ and the corresponding size distribution of them (b), (d) and (f), respectively. Concentration: AuNCs $0.1 \mathrm{mg} \mathrm{mL}^{-1}, \mathrm{Fe}^{3+} 0.1 \mathrm{mM}$, and AA $0.1 \mathrm{mM}$.

surface state of Au in AuNCs by adjustment of the content of $\mathrm{Au}(0)$.

FT-IR. The infrared spectroscopy studies the surface functional groups of AuNCs (Fig. 3c). AuNCs have a typical FT-IR peak at $1737 \mathrm{~cm}^{-1}$ which is from the $\mathrm{COO}^{-}$group. However, the peak at $2524 \mathrm{~cm}^{-1}$ belongs to the -SH of GSH. By comparison, the FT-IR spectrum of AuNCs, the peak of the S-H bond for
GSH disappeared, and then the Au-S bond is formed. Thus, GSH is fixed to the surface of the AuNCs. These results show the successful preparation of the AuNCs.

\section{Optimization of conditions}

To achieve the optimum detection performance, the conditions for synthesis of AuNCs and determination of $\mathrm{Fe}^{3+}$ and $\mathrm{AA}$ were 

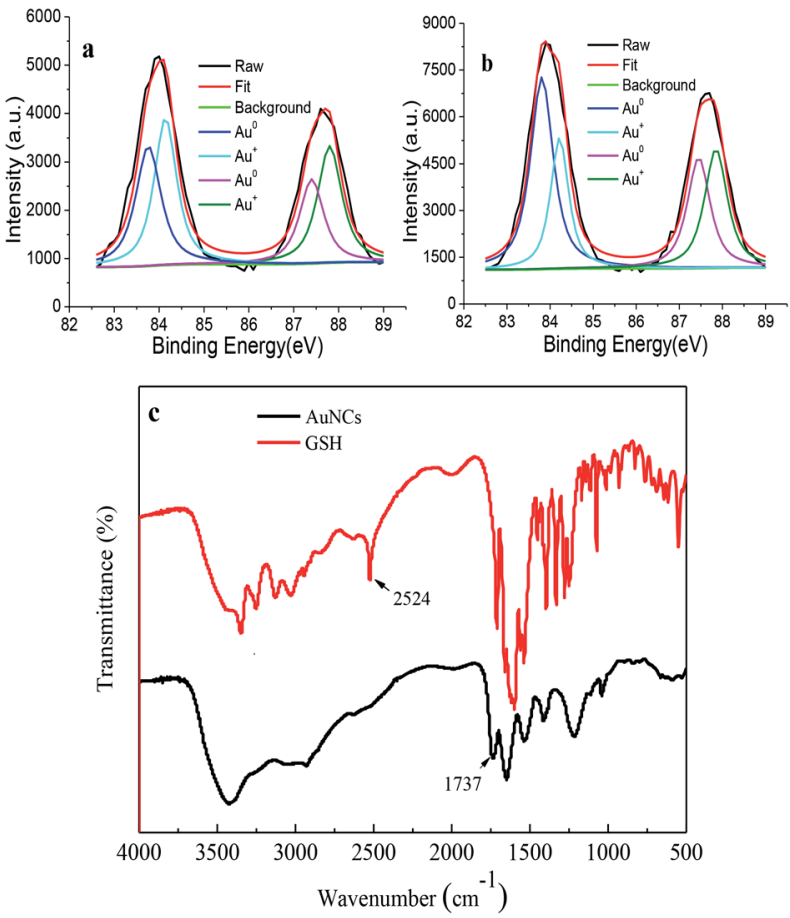

Fig. 3 HR-XPS of Au in the systems of AuNCs (a) and AuNCs + Fe ${ }^{3+}(b)$, and FT-IR spectra of AuNCs and GSH (c). Concentration: AuNCs $0.1 \mathrm{mg} \mathrm{mL}^{-1}, \mathrm{Fe}^{3+} 0.1 \mathrm{mM}$.

studied including reaction time, reaction temperature, buffer species, $\mathrm{pH}$, and ion strength.

\section{Effect of synthesis conditions}

As illustrated in Scheme 1, AuNCs were prepared by a one pot process using $\mathrm{HAuCl}_{4}$ and GSH as precursors with a molar ratio of $2: 3$. The synthesis conditions were investigated by comparing the optical properties of AuNCs, such as the molar ratio of precursors, reaction temperature and hydrothermal time, as shown in Fig. S2 (in ESI $\dagger$ ). Fig. S2a (in ESI $\dagger$ ) shows a fluorescence intensity change with the molar ratio of $\mathrm{HAuCl}_{4}$ to GSH from $2: 1$ to $2: 7$, with the fluorescence intensity reaching its peak at $2: 3$. As shown in Fig. S2b (in ESI $\dagger$ ), as the reaction temperature rises from $40^{\circ} \mathrm{C}$ to $80^{\circ} \mathrm{C}$, the fluorescence intensity of AuNCs increases, while it decreases in the range of 80-100 ${ }^{\circ} \mathrm{C}$. Finally, we study the effect of reaction time on the fluorescence intensity of AuNCs. From 4th hour, the fluorescence intensity is measured every 2 hours. When the intensity varies from 4 to 24 hours, the heating time of the hydrothermal reaction shows a maximum of 16 hours (Fig. S2c, in ESI $\dagger$ ). Therefore, AuNCs were prepared from $\mathrm{HAuCl}_{4}$ and GSH with a series of optimum synthesis conditions including a molar ratio of $2: 3\left(\mathrm{HAuCl}_{4}: \mathrm{GSH}\right)$, heating at $80{ }^{\circ} \mathrm{C}$ for 16 hours.

\section{Effect of analysis conditions}

The effects of $\mathrm{pH}$ and ionic strength were optimized. Fig. S3a (in ESI $\dagger$ ) shows that the fluorescence intensity ratios of AuNCs and AuNCs $-\mathrm{Fe}^{3+}$ are not significantly changed in the $\mathrm{pH}$ range of 212. The results show that AuNCs and AuNCs- $-\mathrm{Fe}^{3+}$ show good stability in a wide $\mathrm{pH}$ range. For the detection of AA, it can be observed that in the solution with $\mathrm{pH}$ varying from 4 to 11 , the fluorescence intensity ratios are relatively stable. Therefore, considering the efficiency and stability of the experiment, ultrapure water with $\mathrm{pH} 6.0$ was used for the determination.

Salt tolerance is one of the most common challenges when conducting nanosensing systems on complex samples. $\mathrm{NaCl}$ was employed to regulate the ionic strength, and the effect of $\mathrm{NaCl}$ concentration on the fluorescence intensity of AuNCs was investigated. As shown in Fig. S3b (in ESI $\dagger$ ), the fluorescence intensity of AuNCs does not change obviously in the concentration range of $0-0.5 \mathrm{M} \mathrm{NaCl}$. It can be concluded that the fluorescence intensity of AuNCs does not change obviously in a wide ionic strength range.

\section{Sensitivity}

Fluorescence titrations are conducted to evaluate the sensitivity of AuNCs for $\mathrm{Fe}^{3+}$ and $\mathrm{AA}$ under optimum conditions. AuNCs
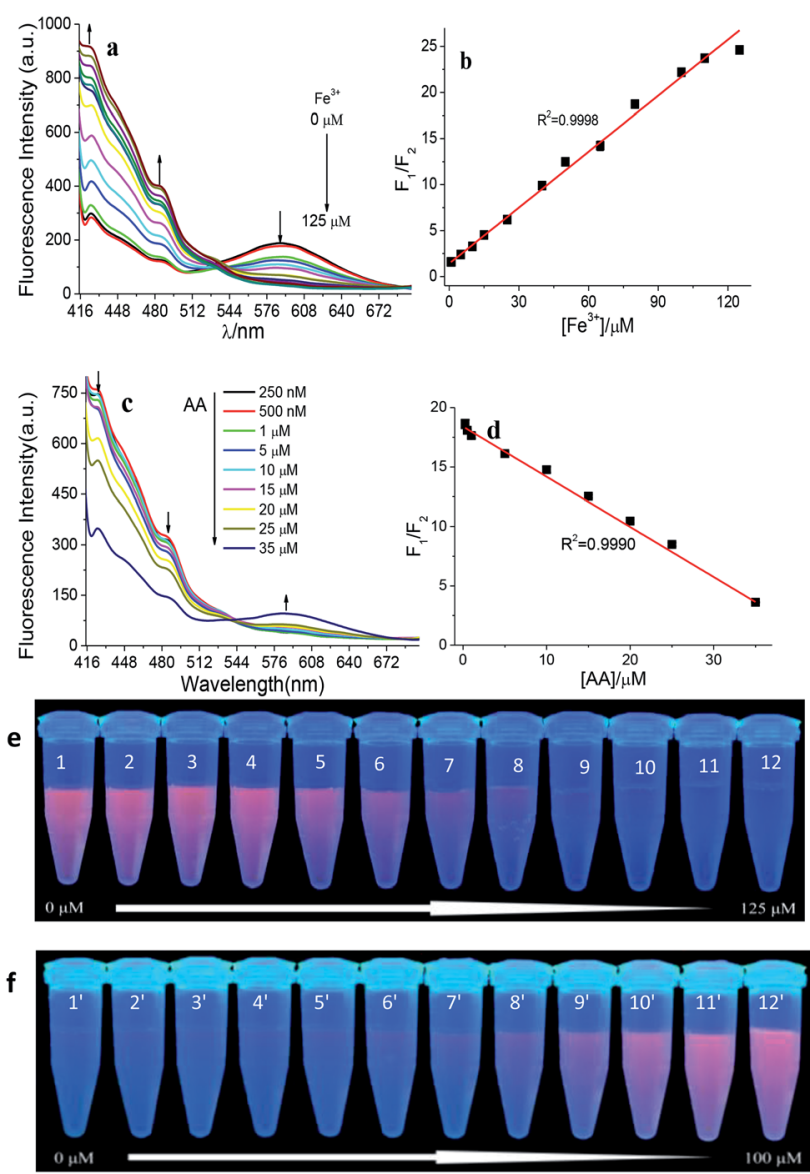

Fig. 4 Fluorescence spectra of AuNCs with and without Fe ${ }^{3+}(a)$ and corresponding linear response of fluorescence ratio to concentration of $\mathrm{Fe}^{3+}(\mathrm{b})$, the fluorescence spectra of AuNCs $+\mathrm{Fe}^{3+}$ with and without AA (c) and corresponding linear response of fluorescence ratio to concentration of AA $(0.25,0.5,1,5,10,15,20,25$, and $35 \mathrm{mM})(\mathrm{d})$, the fluorescence quenching of AuNCs with the increase of $\mathrm{Fe}^{3+}$ concentration can be observed in the UV light (365 nm) (e), and the fluorescence increasing of AuNCs with the increase of $\mathrm{Fe}^{3+}$ concentration can be observed in the UV light $(365 \mathrm{~nm}$ ) (f). Conditions: AuNCs, $0.1 \mathrm{mg} \mathrm{mL}^{-1}, \mathrm{pH} 6.0$, and $25^{\circ} \mathrm{C}$. 
are prepared with the molar ratio of $\mathrm{HAuCl}_{4}: \mathrm{GSH}$ at $2: 3$, heating at $80{ }^{\circ} \mathrm{C}$ for 16 hours and ultrapure water as media with $\mathrm{pH}$ 6.0. In the presence of $\mathrm{Fe}^{3+}(0.75,1.5,10,15,25,40,50,65$, $80,100,110$, and $125 \mu \mathrm{M})$, the fluorescence intensity of AuNCs at $585 \mathrm{~nm}$ was quenched regularly as shown in Fig. 4a, while the fluorescence intensity at $425 \mathrm{~nm}$ increased. Fig. 4b depicts a good linear relationship between the ratio of the fluorescence intensity of the two emission peaks $\left(F_{425} \mathrm{~nm} / F_{585} \mathrm{~nm}\right.$, simplified as $F_{1} / F_{2}$ ) and the concentration of $\mathrm{Fe}^{3+}$ ranges from $0.75 \mu \mathrm{M}$ to $125 \mu \mathrm{M}$ (with a linear correlation coefficient, $\left.r^{2}=0.9998\right)$. The limit of detection (LOD) is $227 \mathrm{nM}$ using $3.29 S_{\mathrm{B}} / m$, rooting in the criterion of the IUPAC recommendation. In this equation, $S_{\mathrm{B}}$ is the standard deviation of the blank $(n=5)$ and $m$ is the slope of the linear calibration curve, respectively. At the same time, the fluorescence quenching of AuNCs with the increase of $\mathrm{Fe}^{3+}$ concentration can be observed in the UV light (365 nm) (Fig. 4e).

Fig. 4c exhibits that the fluorescence peaks of AuNCs at $585 \mathrm{~nm}$ and $425 \mathrm{~nm}$ are recovered gradually, after the addition of AA with different concentrations $(0.25,0.5,1,5,10,15,20,25$, and $35 \mu \mathrm{M}$ ) into AuNCs-Fe ${ }^{3+}$ system. There is a good linear relationship between $F_{1} / F_{2}$ and the concentration of AA in the range of $0.25-30 \mu \mathrm{M}\left(r^{2}=0.9990\right)$ (Fig. $\left.4 \mathrm{~d}\right)$. The LOD is $75.8 \mathrm{nM}$ with the calculation from $3.29 S_{\mathrm{B}} / m$. Then the AuNCs-Fe ${ }^{3+}$ system was established to detect AA sensitively. In addition, the fluorescence recovery of AuNCs ascribed from AA can also be observed from the digital photos under the $365 \mathrm{~nm}$ UV lamp with increase of AA concentration (Fig. 4f).

\section{Specificity and reusability}

Firstly, the selectivity of $\mathrm{Fe}^{3+}$ detection was investigated. A series of AuNCs samples were prepared by adding $100 \mu \mathrm{M} \mathrm{Fe}{ }^{3+}$ or $1 \mathrm{mM}$ other metal ion species. When $\mathrm{Fe}^{3+}$ was added to AuNCs, a drastic change of $F_{1} / F_{2}$ can be obtained in Fig. $5 \mathrm{a}$. There are slight change of $F_{1} / F_{2}$ for other metal ions including $\mathrm{Cu}^{2+}, \mathrm{Hg}^{2+}$, $\mathrm{Zn}^{2+}, \mathrm{Ca}^{2+}, \mathrm{Mg}^{2+}, \mathrm{Pb}^{2+}, \mathrm{Cr}^{3+}, \mathrm{Co}^{2+}, \mathrm{Ba}^{2+}, \mathrm{Cd}^{2+}, \mathrm{Ni}^{2+}$, and $\mathrm{Mn}^{2+}$, even at 10 times higher concentration than that of $\mathrm{Fe}^{3+}$. Furthermore, the specificity for the determination of $\mathrm{Fe}^{3+}$ was also studied with the coexistence of $\mathrm{Fe}^{3+}$ and all other metal ions. The $F_{1} / F_{2}$ value of AuNCs-Fe ${ }^{3+}$ is almost as same as that of AuNCs-Fe ${ }^{3+}$ with other metal ions (Fig. 5b). Therefore, AuNCs can be used as a fluorescence probe for the determination of $\mathrm{Fe}^{3+}$ with high specificity.

The specificity of AA detection was also tested for AuNCs$\mathrm{Fe}^{3+}$ system. Several common biomolecules as common distractors in biological samples were investigated, including urea, UA, Cys, Glu, Pro, Met, Asp, Gly and Mel. Fig. 5c depicts the fluorescence recovery of AuNCs- $\mathrm{Fe}^{3+}$ system in various common biomolecules, indicating that the sensing system has a high selectivity for AA determination. The present method was compared with other reports for $\mathrm{Fe}^{3+}$ and $\mathrm{AA}$ assays listed in Table 1. By comparison, the present method has a wider linear range and a lower detection limit.

Finally, the reusability of AuNCs was carried out for $\mathrm{Fe}^{3+}$ and AA determination, since $\mathrm{Fe}^{3+}$ can quench and AA can recover the fluorescence of AuNCs, respectively. The value of $F_{1} / F_{2}$ for
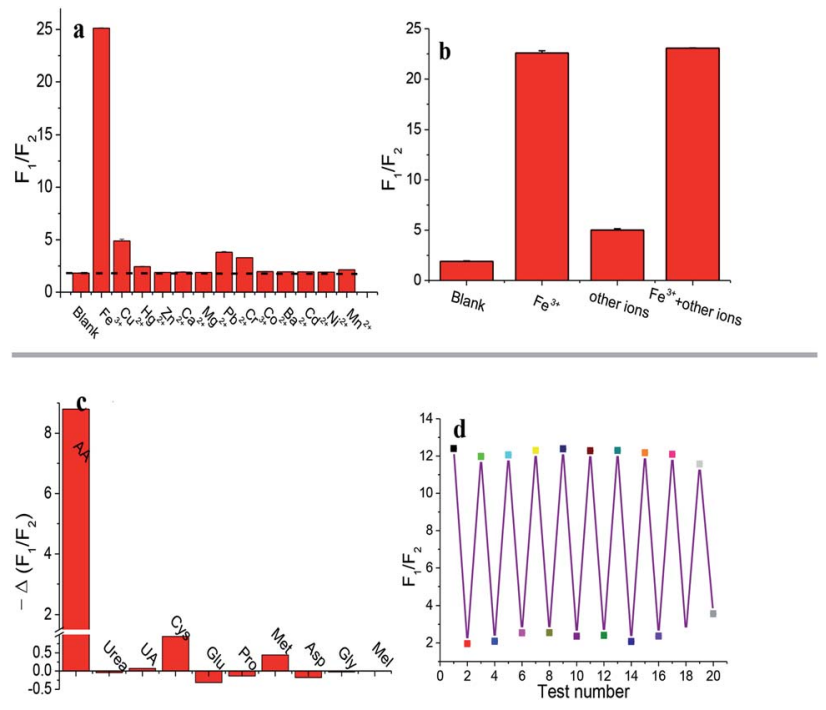

Fig. 5 Effect of metal ions (a) and comparison of metal ions mixture and $\mathrm{Fe}^{3+}$ (b) on the fluorescence ratio of AuNCs, effect of several common biomolecules on the fluorescence ratio of $\mathrm{AuNCs}-\mathrm{Fe}^{3+}$ (c), and cycle times of the AuNCs fluorescence system for $\mathrm{Fe}^{3+}$ and AA (d).

AuNCs can increase with the addition of $\mathrm{Fe}^{3+}$, while decrease in the presence of AA. As Fig. 5d showed, the value of $F_{1} / F_{2}$ for AuNCs- $\mathrm{Fe}^{3+}$ slides down in the presence of AA and goes up with the addition of $\mathrm{Fe}^{3+}$. The change of $F_{1} / F_{2}$ value is basically stable for AuNCs- $\mathrm{Fe}^{3+}$ and AuNCs- $\mathrm{Fe}^{3+}-\mathrm{AA}$ with the test number no more than 17. Thus, the AuNCs fluorescence system can be reused for 8 cycle times.

\section{Analysis of real samples}

To confirm the practical application of the method, a series of water and drug samples were prepared for the determination of $\mathrm{Fe}^{3+}$ and AA. In addition, fetal bovine serum was used as the second true Matrix, so the feasibility of using AuNCs as fluorescence probe to determine $\mathrm{Fe}^{3+}$ in water samples and serum was evaluated. After the sample is pretreated as described in the experiment section, the concentration of $\mathrm{Fe}^{3+}$ was investigated by this method and the National Standard $o$-phenanthroline method, ${ }^{31}$ respectively. It can be seen from Table 2 that the recovery of $\mathrm{Fe}^{3+}$ in three samples is varying from $90.7 \%$ to $106.0 \%$. At the same time, $t$-test results are listed by comparison with the results obtained from the two methods. There was no significant difference between the optimal value and the experimental data with the confidence level of 0.95 . These results confirm the potential of the sensing system for quantitative analysis of $\mathrm{Fe}^{3+}$ in environmental and biological samples. Then, in order to test the practicability of AuNCs- $\mathrm{Fe}^{3+}$ system, it was applied to the determination of AA in tablets. For this purpose, the VC tablets samples were prepared according to the previous work. ${ }^{32}$ AA was determined by this method and $o$ phenylenediamine method. ${ }^{33}$ The results are shown in Table 2. The $t$-test shows that there is no essential difference between the results of our method and those of the national standard method at the confidence level of 0.95 . It is considered that the 
Table 1 Comparison of different PL sensors for sequential determination of $\mathrm{Fe}^{3+}$ and $\mathrm{AA}$

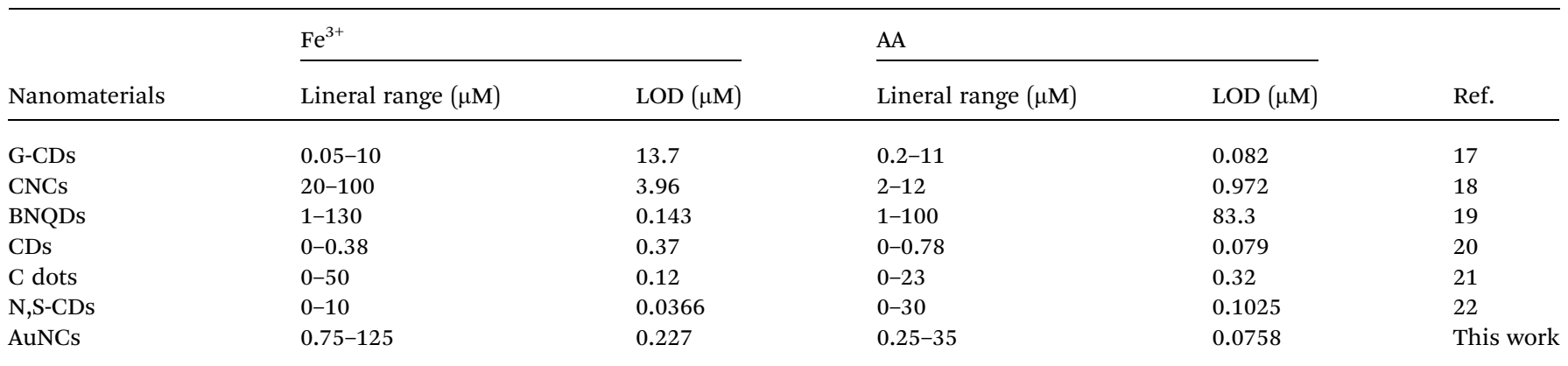

system is feasible for the determination of AA in complex samples.

\section{Possible mechanism for detection of $\mathrm{Fe}^{3+}$ and $\mathrm{AA}$}

As can be seen clearly from Fig. 4a, with the increase of $\mathrm{Fe}^{3+}$ concentration, the fluorescence intensity of AuNCs decreases gradually at $585 \mathrm{~nm}$ and increases sharply at $425 \mathrm{~nm}$. Gradually at $585 \mathrm{~nm}$ and increases sharply at $425 \mathrm{~nm}$.

Due to the surface ligand, the surface motif optimization and reconstruction, the $\mathrm{Au}(\mathrm{I})$-thiolate motif reconstruction determines the dual-emission characteristics of GSH-AuNCs. ${ }^{34}$ Their emission properties show strong dependence on the particle size, the amount of surface $\mathrm{Au}^{+}$(ref. 35) and the surface ligands. ${ }^{35,36}$ The blue emission at $425 \mathrm{~nm}$ is the ligand-metal charge transfer (LMCT) effect from the Au(I)-carboxyl group (the oxygen atom in the carboxylate ligands to the $\mathrm{Au}(\mathrm{I}))$ to the $\mathrm{Au}(0)$ core and subsequent radiative relaxation. ${ }^{35}$ The photoluminescence peak at $585 \mathrm{~nm}$ is the LMCT from the surface of $\mathrm{Au}(\mathrm{I})-\mathrm{GSH}$ motif to the $\mathrm{Au}(0)$ core. The combination of $\mathrm{Fe}^{3+}$ and the carboxyl group has little effect on the charge transfer from the $\mathrm{Au}(\mathrm{I})$-carboxyl group to the $\mathrm{Au}(0)$ core. $\mathrm{Fe}^{3+}$ crosslinks the nanoclusters, and enhances the rigidity of the Au(I)-carboxyl group motif, thus enhances the fluorescence intensity at $425 \mathrm{~nm}$. After the addition of $\mathrm{Fe}^{3+}$, AuNCs has a strong aggregation in the TEM image (Fig. 2). The aggregation of AuNCs is owing to the strong coordination of $\mathrm{Fe}^{3+}$ with $\mathrm{Au}(\mathrm{I})-$ GSH. Since $\mathrm{Fe}^{3+}$ has an electron-withdrawing capacity, the interaction between $\mathrm{Fe}^{3+}$ and the surface of $\mathrm{Au}(\mathrm{I})-\mathrm{GSH}$ motif can reduce the charge transfer effect from the $\mathrm{Au}(\mathrm{I})-\mathrm{GSH}$ motif to the $\mathrm{Au}(0)$ core. And thus the fluorescence intensity at $585 \mathrm{~nm}$ decreases.

To prove the redox reaction between $\mathrm{Fe}^{3+}$ and $\mathrm{AA}$, a series of experiments including spectrophotometry and cyclic voltammetry measurements were carried out (Fig. S1 in ESI $\dagger$ ). The fluorescence spectra (Fig. S1a in ESI $\dagger$ ) illustrates distinctly that $\mathrm{Fe}^{3+}$ quenches the red emission of AuNCs and AA recoveries the AuNCs- $\mathrm{Fe}^{3+}$ fluorescence, respectively. Undoubtedly, $\mathrm{Fe}^{3+}$ and AA don't have fluorescence signal at the AuNCs emission band. Furthermore, the fluorescence change of AuNCs can be strongly ignored induced by AA. So, $\mathrm{Fe}^{3+}$ can only reacted with AA. From absorption spectra (Fig. S1b in ESI $\dagger$ ), it can be distinctly observed that the absorption peak is at 268 and $286 \mathrm{~nm}$ for AA and $\mathrm{Fe}^{3+}$, respectively, while there is no obvious absorption peak for $\mathrm{Fe}^{3+}-\mathrm{AA}$ in the range of $220-600 \mathrm{~nm}$. The reaction of $\mathrm{Fe}^{3+}$ and AA can be demonstrated. Fig. S1c (in ESI $\dagger$ ) depicts the anodic oxidation peak for AA disappears in the $\mathrm{Fe}^{3+}-\mathrm{AA}$ system, and the redox reaction is irreversible for $\mathrm{Fe}^{3+}$ and $\mathrm{AA}$ and in the presence of AuNCs.

Table 2 Results of $\mathrm{Fe}^{3+}$ and AA detection in real samples by the present and reference methods ${ }^{a}$

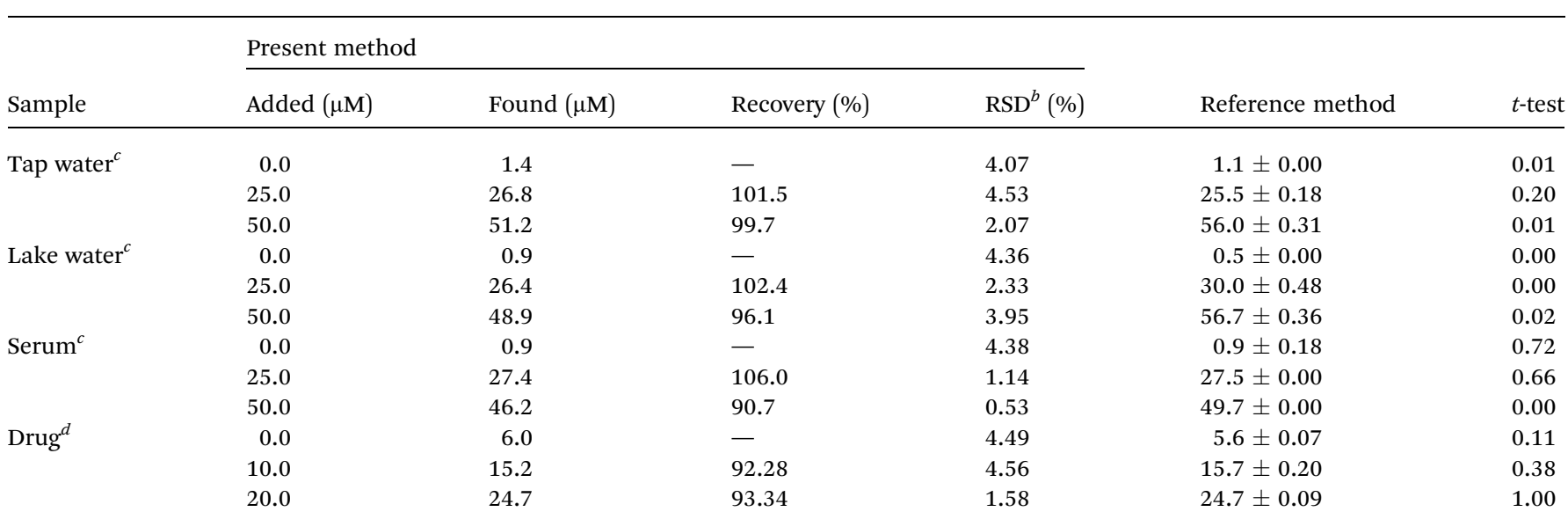

${ }^{a}$ The results were from three separated measurements. ${ }^{b}$ RSD, relative standard deviation. ${ }^{c}$ Samples for Fe ${ }^{3+}$ detection. $^{d}$ Samples for AA detection. 


\section{Conclusions}

In summary, a simple approach was proposed to prepare dualemission AuNCs with GSH as stabilizing reagent. AuNCs show bright pink emission with two fluorescence peaks at 425 and $585 \mathrm{~nm}$, respectively. $\mathrm{Fe}^{3+}$ can quench the fluorescence of AuNCs at $585 \mathrm{~nm}$, while $\mathrm{Fe}^{3+}$ can enhance that at $425 \mathrm{~nm}$. Therefore, a ratiometric fluorescence system is constructed for the determination of $\mathrm{Fe}^{3+}$. In the presence of $\mathrm{AA}$, however, the fluorescence of AuNCs can be recovered due to the interaction between $\mathrm{AA}$ and $\mathrm{Fe}^{3+}$. The redox reaction of $\mathrm{AA}$ and $\mathrm{Fe}^{3+}$ can weaken the effect of $\mathrm{Fe}^{3+}$ on the surface of AuNCs. And then the AuNCs- $\mathrm{Fe}^{3+}$ system is employed for the detection of AA. Comparing with previous works for $\mathrm{Fe}^{3+}$ and $\mathrm{AA}$ assays, the approach exhibits lower LOD. Furthermore, the proposed AuNCs system was successfully used to determine $\mathrm{Fe}^{3+}$ and AA in lake water, human serum, and drug samples. It holds a potential application in the fields related to environmental, biological and pharmaceutical studies.

\section{Conflicts of interest}

There are no conflicts to declare.

\section{Acknowledgements}

This work was supported financially by the Natural Science Foundation of China (91543206), the Natural Science Foundation (ZR2014BQ017, ZR2015BM024, and 2013SJGZ07) and research foundation of Liaocheng University.

\section{Notes and references}

1 L. R. Lynch, Nutr. Rev., 1997, 55, 102-110.

2 C. D. Ankan and R. A. Doong, ACS Appl. Mater. Interfaces, 2016, 8, 21002-21010.

3 J. D. Haas and T. Brownlie, J. Nutr., 2001, 131, 676S-688S.

4 M. Zheng, H. Q. Tan, Z. G. Xie, L. G. Zhang, X. B. Jing and Z. C. Sun, ACS Appl. Mater. Interfaces, 2013, 5, 1078-1083.

5 X. Y. Mu, L. Qi, J. Qiao and H. M. Ma, Anal. Methods, 2014, 6, 6445-6451.

6 D. Wang, C. Chen, X. B. Ke, N. Kang, Y. Q. Shen, Y. L. Liu, H. J. Wang, C. Q. Chen and R. Li, ACS Appl. Mater. Interfaces, 2015, 7, 3030-3040.

7 K. Etsu, N. Yuko and K. Shosuke, Anal. Chem., 1992, 64, 1505-1507.

8 Y. Y. Peng, Y. W. Zhang and J. N. Ye, J. Agric. Food Chem., 2008, 56, 1838-1844.

9 H. J. Cheng, X. Y. Wang and H. Wei, Anal. Chem., 2015, 87, 8889-8895.

10 X. H. Zhu, T. B. Zhao, Z. Nie, Y. Liu and S. Z. Yao, Anal. Chem., 2015, 87, 8524-8530.

11 P. Imagard, P. P. Carolina, C. Eduardo, C. Pablo, L. Susan and S. A. Juan, J. Biol. Chem., 2009, 284, 13306-13315.

12 M. E. Rice, Trends Neurosci., 2000, 23, 209-216.
13 Y. Oscar, C. Julie, D. M. Wong, G. J. Patti, S. R. Antonion, H. B. Paul, S. A. Trauger, C. Desponts, S. Ding and G. Siuzdak, Nat. Chem. Biol., 2010, 6, 411-417.

14 P. Nahid and H. Reza, Anal. Chim. Acta, 2005, 549, 124-128. 15 H. C. Chang, Y. F. Chang, N. C. Fan and J. A. Ho, ACS Appl. Mater. Interfaces, 2014, 6, 18824-18831.

16 B. A. Kairdolf, X. M. Qian and S. M. Nie, Anal. Chem., 2017, 89, 1015-1031.

17 Y. L. Kong, Y. He, J. Zhou, S. H. Zhong and G. W. Song, ChemistrySelect, 2020, 5, 3828.

18 V. Raveendran, A. R. S. Babu and N. K. Renuka, RSC Adv., 2019, 9, 12070.

19 M. Shamsipur, K. Molaei, F. Molaabasi, M. Alipour, N. Alizadeh, S. Hosseinkhani and M. Hosseini, Talanta, 2018, 183, 122.

20 J. Bi, H. Wang, T. Kamal, B.-W. Zhu and M. Tan, RSC Adv., 2017, 7, 30481.

21 Q. X. An, Q. L. Lin, X. H. Huang, R. J. Zhou, X. Guo, W. Z. Xu, S. Y. Wang, D. Xu and H. T. Chang, Dyes Pigm., 2021, 185, 108878.

22 Y. J. Nie, J. Q. Guo, Y. H. Deng and W. Weng, J. Saudi Chem. Soc., 2020, 24, 865-873.

23 Y. X. Jia, X. J. Zhang, C. X. Yin, X. Zhang, J. P. Zhang, X. W. Wang and J. W. Xin, Anal. Methods, 2019, 11, 39743980.

24 X. J. Wu, F. Kong, C. Q. Zhao and S. N. Ding, Analyst, 2019, 144, 2523-2530.

25 L. Wang, H. X. Cao, Y. S. He, C. G. Pan, T. K. Sun, X. Y. Zhang, C. Y. Wang and G. X. Liang, J. Saudi Chem. Soc., 2019, 282, 78-84.

26 M. L. Guo, J. T. Chi, Y. J. Li, G. I. N. Waterhouse, S. Y. Ai, J. Y. Hou and X. Y. Li, Microchim. Acta, 2020, 187, 534.

27 S. Li, P. C. Huang and F. Y. Wu, New J. Chem., 2017, 41, 717723.

28 National food safety standards determination of amino acids in foods, GB5009.124-2016.

29 D. Bain, S. Maity, B. Paramanik and A. Patra, ACS Sustainable Chem. Eng., 2018, 6, 2334-2343.

30 S. C. Zhou, Y. Y. Duan, F. Wang and C. Y. Wang, Nanoscale, 2017, 9, 4981-4988.

31 Water treatment chemicals-general method for determination of iron content, GB/T22596-2008.

32 W. W. Cui, Y. Y. Wang, D. D. Yang and J. X. Du, Microchim. Acta, 2017, 184, 4749-4755.

33 Determination of total ascorbic acid in feeds- $O$ phenylenediamine fluorometry, GB/T17816-1999.

34 X. F. Lou, F. F. Yu, Z. Cao, Y. Xu, L. Yang and H. L. Liu, Chem. Commun., 2020, 56, 7112-7115.

35 Y. T. Chen, T. Q. Yang, H. F. Pan, Y. F. Yuan, L. Chen, M. W. Liu, K. Zhang, S. J. Zhang, P. Wu and J. H. Xu, J. Am. Chem. Soc., 2014, 136, 1686-1689.

36 T. Q. Yang, S. Dai, H. Tan, Y. X. Zong, Y. Y. Liu, J. Q. Chen, K. Zhang, P. Wu, S. J. Zhang, J. H. Xu and Y. Tian, J. Phys. Chem. C, 2019, 123, 18638-18645. 